\title{
Brain science and human culture
}

\author{
Alfredo Margreth · Ernesto Carafoli • \\ Giovanni Berlucchi
}

Received: 2 July 2014/ Accepted: 3 July 2014/Published online: 24 July 2014

(C) Accademia Nazionale dei Lincei 2014

Human beings are believed to be the only animals who have superimposed on their natural evolution an evolution of their own making, thus begetting human history as well as human culture in all its ramifications. The possession of language, a symbolic way of processing and exchanging information about the world, confers on humans a unique cognitive style which sets them apart from all other animals. Because of language, humans are endowed with an ability for mental time travel and with an inherent disposition to inquire about their place in the universe and more generally about the nature of their humanness. For centuries scholars of various humanistic disciplines have looked into the human mind, at both the individual and societal levels, for providing a cogent answer to the question of what makes us human. Nowadays, the advances of neuroscience in revealing the neural bases of mental processes have shown that the answer cannot be exhaustive without looking into the human brain. We are human because we have a human brain, shaped by the genes but continuously modified and moulded by experience throughout our entire life; and each of us, including the so-called identical twins, is unique because each human brain is different from all other human brains. Contemporary neuroscience has the

\footnotetext{
A. Margreth

Accademia Nazionale dei Lincei, Rome, Italy

e-mail: alfredo.margreth@alice.it

E. Carafoli

Venetian Institute of Molecular Medicine, Padua, Italy

e-mail: ernesto.carafoli@unipd.it

G. Berlucchi $(\square)$

Department of Neurological and Movement Sciences, University

of Verona, Verona, Italy

e-mail: giovanni.berlucchi@univr.it
}

ambition to disclose how our brains determine who we are and how we socially interact and share with others the systems of values, beliefs and knowledge which are part of any human culture. In addition to having the potential for a greater understanding of ourselves as human beings, the facts, ideas, concepts and images of neuroscience are now the source of fresh perspectives in many sectors of learning, culture and society, from the humanities to the social sciences, from literature to the figurative arts, from architecture to the theatre and the cinema. That all human activities depend on the functioning of the brain is an obvious truism, but one truism that has long been neglected because of an apparently inborn metaphysical dualism typical of human beings. The flourishing of research fields denoted by the neuro prefix, such as neuroaesthetics, neuroethics, neuroeducation, neuroeconomy, neuromarketing and many others, shows that probing the brain correlates of forms of cognition and behaviour specific to these fields has now come to be regarded as important or even essential for their full comprehension and representation. The identification of humans as cerebral subjects is seen by many as a hallmark of modernity, and the pervasive influence of neuroscience knowledge on current ways of thinking justifies the widespread belief that we are now living in a neuroculture. Within neuroscience itself a specific interdisciplinary sector has developed, called cultural neuroscience, that aims at investigating the relationships between culture and human brain functions.

The articles in the present issue of Rendiconti Lincei provide some select examples of the integration of neuroscience and traditional humanistic thought into the socalled neuroculture and neurospecialties. The articles by Rolls, Cela Conde et al., Rizzolatti, and Jäncke discuss material that was presented at the Accademia dei Lincei in Rome during the Golgi Day Symposium on "Brain Science 
and Human Culture" held on June 3, 2013. The articles by Schettino and Schurrer are invited reviews which are included in the Issue as their contents are related to the topics discussed in the Symposium.

In their article, Cela Conde et al. describe how the new technological means for the noninvasive visualization of human brain activities have led to the detailed knowledge of the neural correlates of the perception of beauty, suggesting that evolutionary changes in human frontal and parietal areas can account for human aesthetic appreciation and even artistic creation.

Rizzolatti's article argues (admittedly in a speculative way) that a selected-for increase in the number of mirror neurons at some point in the history of Homo sapiens may have been crucial, through an essential process of imitation, for the construction of internal and private experiences and for developing the awareness that other beings undergo similar internal and private experiences. The number of mirror neurons in monkeys and chimps would only be sufficient for the emulation of actions of others, but not for the process of copying and identifying with the purposeful behaviours of others which is believed to underlie the emergence of human culture.

Rolls' article furnishes a wide-ranging rationale for the existence of the new neurospecialties (neuroethics, neuroaesthetics, neuroeconomics, neuroreligion and so forth) by arguing that basic neural emotional mechanisms may be at the roots of everyday human experiences and behaviours such as moral choices, decision-making in the court, aesthetic appreciation, sexual preferences, religious beliefs, etc.

Jäncke'paper provides a survey of the phenomena and possible mechanisms of the phenomenon of synesthesia, which is the occurrence of a sensory experience in one modality when a stimulus is delivered to another modality. The experimental evidence supports the notion that the synesthetic brain may be peculiarly wired up, or at least that some intermodal connections may enjoy a hyperfunctional potentiation. The possibility that synesthesia endows its possessors with an evolutionary benefit, and the relationships between synesthesia and artistic talents are also discussed in the paper.

The reviews by Schummer and Schettino deal with the relationships between aesthetics and chemistry from different points of view. They do not mention neuroscience directly, but implicitly suggest that the appreciation of different kinds of beauty could well rely on the same biological (i.e. cerebral) mechanisms. Schummer argues that aesthetic values afford important motives and guidance in chemical research. By excluding epistemic, instrumental, and ethical values from the definition of aesthetics, he describes various aesthetic facets of chemistry, from the sensual qualities of materials to the beauty of molecular representation, from the artistic finesse of some chemical experiments to the elegance of mathematical modelling in chemical engineering and physical chemistry. Schettino's review focuses on the role of language in chemical description and uses citations from Lucretius, Goethe, Primo Levi, Italo Calvino and others to show that chemistry and the chemical lexicon have several points of contact and affinities with the fine arts and literature. The sharing of some principles of design by beautifully symmetric chemical molecules, beautiful buildings and beautiful paintings hint at some basic underlying mechanism which can only reside in the human brain. 